\title{
Caracterización de la lana de ovejas Criollas argentinas en cuatro ambientes diferentes
}

Peña, S. ${ }^{1}$; Sacchero, D. ${ }^{2}$; Maurino, J. ${ }^{2}$; López, G.A. ${ }^{\text {; }}$ Abbiati, N.N. ${ }^{1}$; Género, E.R. ${ }^{1}$ y Martínez, R.D. ${ }^{1}$

'Facultad de Ciencias Agrarias. UNLZ. Buenos Aires. Argentina.

${ }^{2}$ Laboratorio de Fibras Textiles. INTA. Bariloche. Argentina.

PaLABRAS CLAVE ADICIONALES

Ovinos Criollos.

Fibras.

Calidad.

Características.

\section{RESUMEN}

La raza ovina Criolla es la fundadora del patrimonio ovino argentino y está distribuida en casi todas las provincias. Son pocos los trabajos destinados a estudiar la calidad lanera de este germoplasma. El objetivo fue caracterizar la lana de ovejas Criollas Argentinas, utilizando las principales medidas que determinan su calidad y su valor comercial. El muestreo se realizó sobre majadas ubicadas en cuatro provincias: Salta $(S A)(n=44)$; Santiago del Estero (SE) $(n=60)$; Corrientes (CO) $(n=40)$ y Buenos Aires (BA) $(n=59)$. Se tomaron muestras de lana de cada oveja y se determinaron las siguientes variables: diámetro medio de fibra (DMF); desvío estándar del diámetro medio de fibra (DE_DMF); curvatura de ondulación (CU); factor de confort (FC) y largo de mecha (LM). El análisis estadístico incluyó análisis de varianzas (ANOVA), análisis de componentes principales (ACP) y de conglomerados (ACO). Las ovejas $\mathrm{SA}$ se destacaron del resto de las regiones por su menor $\mathrm{DMF}=26,1 \pm 2,5 \mu \mathrm{m}$ y $D E \_D M F=6,2 \pm 1,5 \mu \mathrm{m}$, su mayor $\mathrm{CU}=78,5 \pm 13,5 \% \mathrm{~mm}$ y su mayor $\mathrm{FC}=78,8 \pm 12,7 \%$, mientras que las de $B A$ presentaron un $L M=123,0 \pm 36,6 \mathrm{~mm}$, ampliamente superior a las otras regiones y $\mathrm{CO}$ presentó mayor $\mathrm{DMF}=35,9 \pm 4,1 \mu \mathrm{m}$. El ACP identificó 2 componentes que explicaron el $90 \%$ de la variabilidad. La primera diferencia SA de BA y muestra superposición entre SE y CO. La segunda diferencia BA del resto de las regiones. Del ACO surge que los ovinos de SE y CO forman un solo grupo definiéndose tres regiones: SA, BA y SE-CO. Ninguno de los tres grupos respondió a la categoría carpet-wool o Criola correspondiente a la clasificación lanera oficial, por eso se considera necesario construir un mapa lanero Criollo de la República Argentina.

\section{Characterization of the argentine Creole sheep wool in four different environments}

\section{SUMMARY}

The Creole sheep breed is the founder of the argentine sheep heritage and it widespreads through almost all the provinces. There are few studies aimed at assessing this germplasm's wool quality. The objective was to characterize the Creole Argentinean sheep's wool, by using the main measures that determine its quality and commercial value. The sampling concerned flocks located in all the four provinces: Salta (SA) $(n=44)$; Santiago del Estero (SE) $(n=60)$; Corrientes $(C O)(n=40)$ and Buenos Aires $(B A)(n=59)$. Wool samples were taken from each sheep and the following variables were determined: mean fibre diameter (DMF); standard deviation of the mean fibre diameter (DE DMF); mean fibre curvature (CU); comfort factor (FC) and staple length (LM). The statistical analysis included analysis of variance (ANOVA), principal components analysis (PCA) and cluster analysis (CA). The sheep from SA stood out from the ones from the rest of the regions because of their low $D M F=26,1 \pm 2,5 \mu \mathrm{m}$ and $D E \_D M F=6.2 \pm 1.5 \mu \mathrm{m}$, their higher $\mathrm{CU}=78.5 \pm 13.5 \% \mathrm{~mm}$ and $\mathrm{FC}=78.8 \pm 12.7 \%$, while the ones from $B A$ presented a $L M=123.0 \pm 36.6 \mathrm{~mm}$ value, which was remarkably higher than the ones from the other regions and $C O$, which showed a higher $D M F=35.9 \pm 4.1 \mu$. The PCA highlighted two components that explained the $90 \%$ of the variability. The first one distinguishes SA from BA and shows the existing overlap between SE and CO. The second one differentiates $B A$ from the rest of the regions. From the $C A$, it can be concluded that the sheep from SE and CO formed only one group, what defined three regions: SA, BA and SE-CO. None of the three regions responds to the carpet-wool o Criolla category according to the official wool classification, for that reason, it is necessary to build a Creole wool map for the Argentine Republic.
Cronología del artículo.

Recibido/Received: 26.03.2015

Aceptado/Accepted: 05.11.2015

On-line: 16.03.2016

Correspondencia a los autores/Contact e-mail:

sabp03@yahoo.com.ar

\section{INTRODUCCIÓN}

La raza ovina Criolla ocupa un destacado lugar en el patrimonio ovino argentino actual. Está distribuida en casi todas las provincias y ocupa el tercer lugar en número de animales, luego de la Merino y la Corriedale con un $8 \%$ de las existencias totales (Mueller, 2006).
Es la raza fundadora de la producción ovina de Argentina, sus primeros ingresos se produjeron a fines del siglo XVI con la llegada de los españoles principalmente por el Perú, por ejemplo en 1587 Juan Torres de Vera y Aragón trajo por esta vía 4000 ovejas que fueron diseminadas entre las provincias de Buenos Aires, Santa Fe y Corrientes (Zeballos, 1898). En 1810 en Argentina 


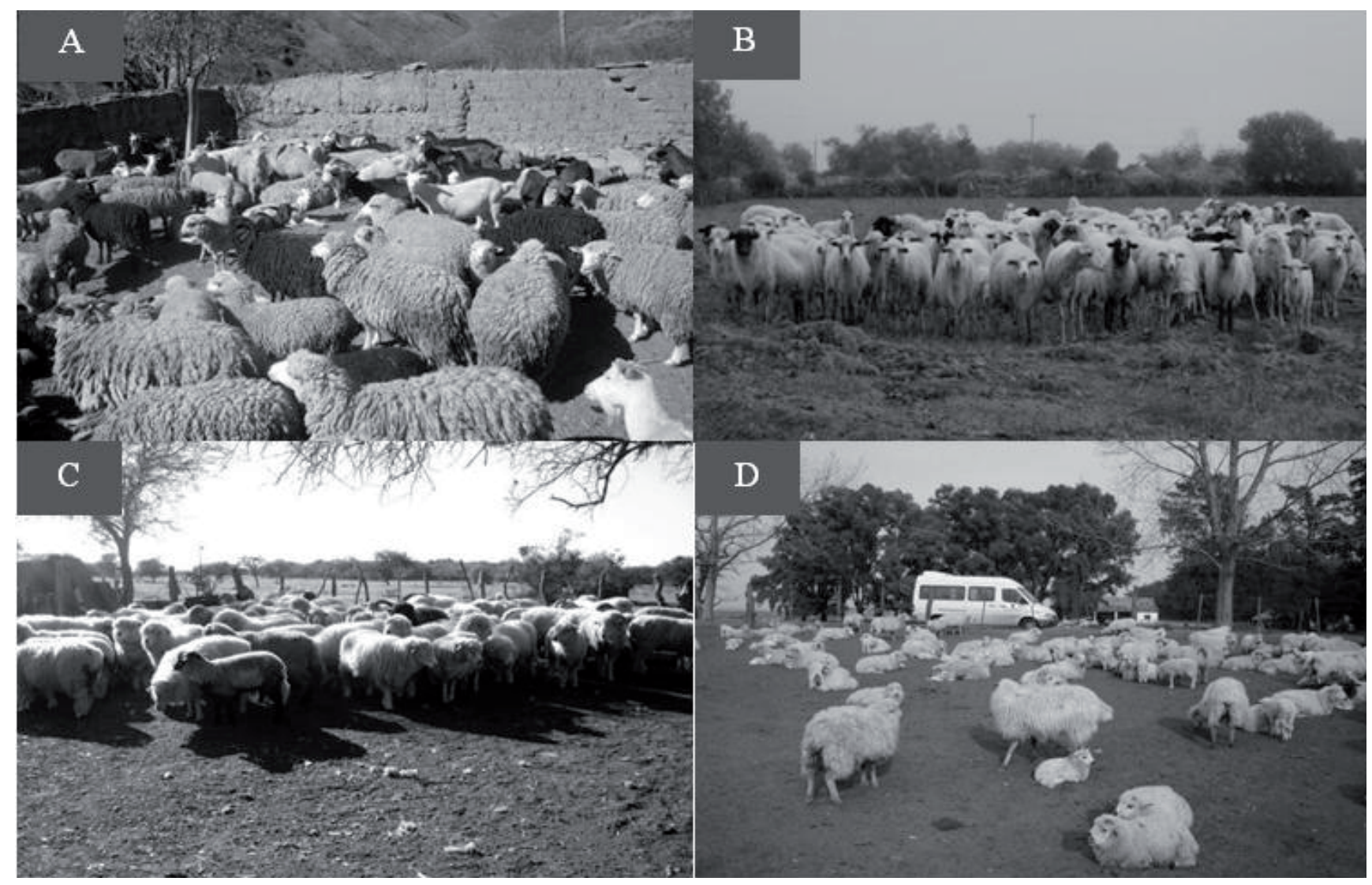

Figura 1. Majadas de ovejas criollas en diferentes regiones de Argentina. A: Iruya, provincia de Salta (SA). B: Departamento Figueroa, provincia de Santiago del Estero (SE). C: Sauce, provincia de Corrientes (CO). D: 25 de Mayo, provincia de Buenos Aires (BA) (Creole herds of sheep in different regions of Argentina. A: Iruya, province of Salta (SA). B: Figueroa department, province of Santiago del Estero (SE). C: Sauce, province of Corrientes (CO). D: May 25, province Buenos Aires (BA)).

ya existían tres millones de lanares de raza Criolla, de cuerpo menudo y lana de distintos colores (Helman, 1951). Fueron muy importantes para la formación y el desarrollo de las primeras poblaciones debido a su facilidad para arrearlos y a su bajo costo utilizándose su lana y su cuero como pellones para montar pero también fueron muy apreciados por los indígenas, quienes lograron mejorar su calidad lanera como los tehuelches septentrionales (Carrazzoni, 1997). En 1825 se realizaron en Buenos Aires los primeros cruzamientos de las ovejas Criollas con Merinos puros importados (Zeballos, 1898). A partir de allí se introdujeron al país diferentes grupos de ovinos de razas españolas e inglesas, con el propósito de mejorar la producción lanera nacional, que se afianzaba por la demanda de lanas finas para satisfacer las industrias europeas durante las décadas de 1830 y 1840 (Carrazzoni, 1997). Este proceso de mestización se intensificó con el tiempo, principalmente en las majadas más numerosas, siendo absorbido paulatinamente el ovino Criollo por las razas introducidas. Sin embargo, debido a su adaptación a los distintos ambientes de nuestro país y a las ventajas productivas valoradas por los pequeños y medianos productores locales de las distintas regiones, el ovino Criollo ha permanecido hasta la actualidad.

Han sido muy pocos los trabajos de investigación desarrollados para estudiar la calidad lanera de este germoplasma, en este sentido pueden mencionarse los resultados obtenidos por Reising et al. (2008) sobre la oveja Linca en el noroeste de la Patagonia y por Galdá- mez et al. (2012), sobre la oveja autóctona de Formosa. La fibra de lana ovina tiene dos destinos principales: 1) el uso industrial donde su valor comercial se determina principalmente por el diámetro medio de fibra y donde también se requieren fibras de color blanco, siendo castigada la presencia de fibras coloreadas (Aguirre et al., 2010) y 2) el uso artesanal donde las características que definen una mejor calidad varían según la región, por ejemplo en el Borrego de Chiapas las mujeres indígenas establecen como prioridad para definir la calidad de la lana, el largo de la misma, el grosor y el color negro (Pérezgrovas Garza y Parés-Casanova, 2013) y las ovejas Criollas norteamericanas de raza Navajo-Churro que son valoradas para la producción de tejidos regionales, famosos en los EEUU por sus cualidades únicas y su pertinencia cultural, tienen variedad de colores (blanco, negro, distintas tonalidades de grises y marrones) (Sponenberg y Taylor, 2009). También en la oveja Linca del noroeste patagónico las hilanderas mapuches valoran el largo de mecha, la suavidad y la diversidad de colores (Reising et al. 2008), es decir que en el caso de los tejidos artesanales se tiende a conservar la variabilidad de las fibras ovinas, mientras que para el uso industrial se tiende a la uniformidad. La lana Criolla se ha clasificado como carpet wool o lana destinada únicamente para la confección de alfombras (Calvo, 1983; Aguirre et al., 2010), sin embargo, la misma puede utilizarse para la confección de diversas prendas como ponchos, chales, bolsos, suéteres, gorros, calcetines, guantes, fajas, billeteras con todo tipo de 
diseño y colorido tal como ocurre en Rio Negro y Chubut a partir de la oveja Linca (Cardinaletti et al., 2011), o en el Centro-Oeste formoseño donde 1200 mujeres artesanas aprovechan la lana de la oveja Criolla para fabricar distintos productos (Galdámez et al., 2012). La producción de tejidos artesanales mejora la economía de diversas regiones, promueve la integración social y tiende al mantenimiento de la diversidad genética ovina, por lo cual se considera que es muy importante conocer las características laneras de las ovejas Criollas adaptadas a las distintas regiones argentinas. El objetivo del trabajo es caracterizar la lana de ovejas Criollas argentinas adaptadas a cuatro ambientes distintos, teniendo en cuenta las principales medidas utilizadas para determinar la calidad de la lana y su valor comercial desde el punto de vista industrial.

\section{MATERIAL Y MÉTODOS}

\section{LOCALIZACIÓN DEL ESTUDIO Y ANIMALES EXPERIMENTALES}

La raza ovina Criolla Argentina no cuenta actualmente con registros genealógicos formales, ni con una asociación de criadores que avale o certifique la pertenencia de los animales a esa raza, por lo cual para identificar los grupos a estudiar y evitar cometer errores en el muestreo, se han tenido en cuenta las siguientes características: la información proporcionada por sus criadores; el aislamiento reproductivo (disminuye la probabilidad de mestización) y tamaño de la majada (permite realizar un muestreo más representativo). La información de los criadores fue la principal herramienta en la definición y elección de los grupos a estudiar. Ellos conocen el origen y la historia evolutiva de sus majadas y saben determinar con precisión cual oveja es Criolla pura y cual es mestiza. Se realizó un trabajo de búsqueda recorriendo la mayoría de las provincias argentinas, contactando con distintos tipos de criadores y técnicos regionales que fueron proporcionando la información necesaria para la elección de las majadas Criollas. Los motivos de descarte para la conformación de los grupos de estudio fueron principalmente: a.) introducción a la majada de algún reproductor de otra raza; b.) la no presencia en la majada de carneros Criollos; c.) desconocimiento por parte del criador del origen de la majada. Por sus antecedentes, características reproductivas y diversidad de ambientes decidimos muestrear animales ubicados en cuatro provincias: Salta (SA) que corresponde a tres majadas ubicadas en las localidades de Iruya y Pueblo Viejo perteneciente a familias de pueblos originarios collas (2800 msnm); Santiago del Estero (SE), también concierne a tres majadas de pequeños productores ubicados en el Departamento Figueroa; Corrientes (CO) se realizó sobre una majada de aproximadamente 200 ovejas de un núcleo cerrado y mantenido como reserva por un criador de la zona de Sauce y por último Buenos Aires (BA) sobre una majada numerosa (aproximadamente 800 ovejas) que se mantienen en núcleo cerrado en la localidad de 25 de Mayo en la estancia La Juanita.

Se tomaron muestras de lana del costillar izquierdo de 203 hembras adultas (considerando su cronometría dentaria), con un período medio de crecimiento de la lana de $330 \pm 18$ días, pertenecientes a los grupos mencionados: $\mathrm{SA}=44 ; \mathrm{SE}=60 ; \mathrm{CO}=40$ y $\mathrm{BA}=59$ (figura 1).

\section{DETERMINACIÓN DE LAS CARACTERÍSTICAS DE LA CALIDAD DE LA LANA}

Las muestras de cada oveja, fueron identificadas y acondicionadas adecuadamente y se enviaron al Laboratorio de Fibras Textiles del INTA Bariloche. Las muestras se analizaron utilizando un equipo OFDA2000®. De las muestras de lana se extraen mechas, las cuales son desengrasadas mediante un baño ultrasónico anexo al equipo en una solución de hexano/isopropílico (80:20) durante 60 segundos. Las mechas desengrasadas se colocan en una gradícula ad hoc y se realizan las mediciones de diámetros en secciones transversales de las mechas de lana cada $5 \mathrm{~mm}$ hasta recorrer toda su longitud. Existe abundante información al respecto (Baxter, 2001a; Baxter, 2001b; Peterson y Gherardi, 2001). El equipo provee los valores de diámetro medio de fibra (DMF; $\mu \mathrm{m}$ ) es una medida del grosor de las fibras individuales, es el principal determinante del precio y calidad de la lana y determina el destino y uso final en la industria; el desvío estándar del diámetro medio de fibra (DE_DMF; $\mu \mathrm{m})$, es un medida de dispersión de las mediciones individuales mencionadas anteriormente; factor de confort (FC; \%) que representa el porcentaje del total de las fibras que tiene menos de 30 micrones, su importancia radica en la estimulación nerviosa que producen sobre la piel las fibras gruesas (picazón), la curvatura media de la ondulación (CU; \% / mm) es una medida usada para describir la ocupación de espacios de una masa de fibras, relevante tanto para prendas de vestir como para tapicería y alfombras; y largo de mecha (LM; mm) es una medida de la longitud de las mechas sobre su eje longitudinal sin estiramientos, que influye sobre su capacidad de procesamiento textil.

\section{ANÁLISIS ESTADÍSTICO}

Se efectuaron análisis descriptivos univariados y de correlación de Pearson, previo análisis de los supuestos. Además, se emplearon las técnicas de análisis multivariado de análisis de componentes principales (ACP) y análisis de conglomerados (AC), (Cuadras, 2014; Peña, 2002). Los resultados del ACP se grafican en un biplot que permite la representación en dos dimensiones (primera y segunda componente principal) de los ovinos y variables medidas en simultáneo (Cuadras, 2014). Para AC se empleó la matriz de distancias euclídeas; la selección del método jerárquico de agrupamiento se realizó a través del coeficiente de correlación cofenética (Sokal y Rohlf, 1962); el número de conglomerados se determinó mediante el criterio de conglomerado cúbico (CCC) y el valor pseudo F (SAS Institute Inc., 2009). También se realizaron análisis de varianzas (ANVA) de cada variable bajo estudio, previo análisis de los supuestos. Cuando no se cumplió el supuesto de homogeneidad de varianzas, se emplearon modelos mixtos con una matriz de errores diagonal heterogénea según región, en el ANVA (Mc Culloch y Searle, 2001). Se emplearon las pruebas de comparaciones múltiples de Tukey (varianzas homogéneas) o Tukey-Cramer (varianzas heterogéneas). Se efectúo un análisis discriminante a los efectos de estudiar el grado 
Tabla I. Valores medios y desvíos estándar para las cinco variables en cada una de las cuatro regiones (Mean values and standard deviations for the five variables in every one of the four regions).

\begin{tabular}{lcccc}
\hline \multicolumn{5}{c}{ Región } \\
\hline Variable & SA $(n=44)$ & SE $(n=60)$ & BA $(n=59)$ & CO $(n=40)$ \\
\hline DMF $(\mu m)$ & $26,1 \pm 2,5^{\text {a }}$ & $33,1 \pm 3,8^{\mathrm{b}}$ & $33,5 \pm 2,9^{\mathrm{b}}$ & $35,9 \pm 4,1^{\mathrm{c}}$ \\
DE_DMF $(\mu \mathrm{m})$ & $6,2 \pm 1,5^{\mathrm{a}}$ & $9,0 \pm 2,5^{\mathrm{b}}$ & $11,2 \pm 2,2^{\mathrm{c}}$ & $9,4 \pm 2,1^{\mathrm{b}}$ \\
CU $(\% \mathrm{~mm})$ & $78,5 \pm 13.5^{\mathrm{a}}$ & $45,5 \pm 15,0^{\mathrm{b}}$ & $41,9 \pm 7,3^{\mathrm{b}}$ & $42,7 \pm 12,1^{\mathrm{b}}$ \\
FC $(\%)$ & $78,8 \pm 12,7^{\mathrm{a}}$ & $47,5 \pm 16,2^{\mathrm{b}}$ & $49,2 \pm 13,8^{\mathrm{b}}$ & $34,0 \pm 16,2^{\mathrm{c}}$ \\
LM $(\mathrm{mm})$ & $49,2 \pm 14,4^{\mathrm{a}}$ & $43,3 \pm 15,8^{\mathrm{a}}$ & $123,0 \pm 36,6^{\mathrm{b}}$ & $58,9 \pm 14,2^{\mathrm{c}}$
\end{tabular}

$\mathrm{SA}=\mathrm{Salta} ; \mathrm{SE}=$ Santiago del $\mathrm{Estero} ; \mathrm{BA}=$ Buenos Aires $; \mathrm{CO}=$ Corrientes; $\mathrm{DMF}=$ Diámetro medio de fibra; $\mathrm{DE}$ DMF= Desvío estándar del diámetro medio de fibra; $\mathrm{CU}=$ Curvatura de ondulación; $\mathrm{FC}=$ Factor de confort; LM= Largo de mecha. Medias en la misma fila con la misma letra no difieren, prueba de Tukey $(p<0,05)$.

Tabla II. Matriz de correlaciones entre variables (Correlation matrix between variables).

\begin{tabular}{lccccc}
\hline & DMF & DE DMF & CU & FC & LM \\
\hline DMF & 1 & & & & \\
DE_DMF & $0,70^{*}$ & 1 & & & \\
CU & $-0,82^{*}$ & $-0,71^{*}$ & 1 & & \\
FC & $-0,95^{*}$ & $0,52^{*}$ & $0,77^{*}$ & 1 & \\
LM & $0,23^{*}$ & $0,60^{*}$ & $-0,33^{*}$ & $-0,10$ & 1 \\
\hline
\end{tabular}

$\mathrm{DMF}=$ Diámetro medio de fibra; DE_DMF= Desvío estándar del diámetro medio de fibra; $\mathrm{CU}=$ Curvatura de ondulación; $\mathrm{FC}=$ Factor de confort; LM= Largo de mecha. ${ }^{*}(p<0,05)$.

Tabla III. Valores propios y proporción de varianza explicada por cada componente principal (CP) (Eigenvalues and variance proportion explained by every principal component (CP)).

\begin{tabular}{cccc}
\hline CP & Valor propio & $\begin{array}{c}\text { Proporción } \\
\text { explicada }\end{array}$ & $\begin{array}{c}\text { Proporción } \\
\text { acumulada }\end{array}$ \\
\hline 1 & 3,41 & 0,68 & 0,68 \\
2 & 1,10 & 0,22 & 0,90 \\
3 & 0,26 & 0,05 & 0,95 \\
\hline
\end{tabular}

de diferenciación entre las regiones a través de la tasa de error por validación cruzada (Cuadras, 2014; Peña, 2002). Se trabajó con un nivel de significación del $5 \%$. Se emplearon los software InfoStat (Di Rienzo et al., 2012) y SAS (SAS Institute Inc., 2009).

\section{RESULTADOS Y DISCUSIÓN}

En la tabla I se presentan los estadísticos descriptivos, medias y desvíos estándar y resultados de las pruebas de Tukey, según región. Se destacan claramente las ovejas salteñas del resto de las regiones por su menor DMF y DE_DMF, su mayor CU y FC, mientras que las de Buenos Aires presentan un LM ampliamente superior a las otras regiones y $\mathrm{CO}$ presenta mayor media en DMF.

La tabla II muestra las correlaciones fenotípicas, donde puede observarse que el DMF está asociado con las restantes variables. En particular, se correlaciona negativamente con FC ( $\mathrm{r}=-0,95)$, lo cual es lógico ya que el FC es mayor para valores pequeños de DMF, en particular menores de $30 \mu \mathrm{m}$ (Sacchero, 2008). También DMF se correlaciona negativamente con $\mathrm{CU}(\mathrm{r}=-0,82)$, es decir que las fibras más finas (menor DMF) tienen mayor CU, coincidiendo con los valores registrados por Guzmán Barzola y Aliaga Gutiérrez (2010) $(\mathrm{r}=-0,32)$ y por Pickering et al. (2013) $(\mathrm{r}=-0,55)$. Por otro lado, DMF y DE_DMF se correlacionan positivamente $(r=0,70)$, con valor similar a $0,64 \pm 0,02$ obtenido en Nueva Zelanda para cuatro razas doble propósito Pickering et al. (2013) e inferior, pero de igual signo y significado estadístico que el valor obtenido por Safari et al. (2005) $(\mathrm{r}=0,40)$. La CU mostró una correlación positiva con FC $(r=0,77)$, lo cual también resulta lógico si consideramos que las fibras con mayor $\mathrm{CU}$ tienen menor DMF. Entre el LM y el DMF la correlación resultó positiva, pero baja $(\mathrm{r}=0,23)$, concordante con los valores obtenidos por Pickering et al. (2013) ( $\mathrm{r}=0,21)$, Safari et al. $(2005)(\mathrm{r}=0,19)$ y Vaillemans $(2003)(\mathrm{r}=0,17)$. Otros autores han reportado correlaciones bajas como Steinhagen et al. (1986) en la raza Dohne Merino $(\mathrm{r}=-0,12)$ y en la raza Merino $(r=-0,15)$, o Guzmán Barzola y Aliaga Gutiérrez (2010) en ovinos Corriedale $(\mathrm{r}=-0,004)$. En otro trabajo Lembeye et al. (2014), calcularon en la raza Merino Precoz una correlación entre LM y DMF de $0,035 \pm 0,10$; pareciera que entre ambas características no existe una influencia mutua (Guzmán Barzola y Aliaga Gutiérrez, 2010).

El ACP permitió identificar 2 componentes principales (CP) (tabla III) que explicaron el 90\% de la variabilidad de los datos. Las correlaciones entre las variables medidas y las CP (tabla IV) muestran como la primera está asociada a la calidad de la fibra para uso industrial (estadísticos asociados a diámetro, curvatura y confort) y la segunda $\mathrm{CP}$ a su largo, relacionado principalmente a las preferencias de uso artesanal. La primera CP diferencia SA (el grupo menos variable) de BA y muestra alta superposición entre SE y CO. La segunda $\mathrm{CP}$ diferencia $\mathrm{BA}$ del resto de las regiones (figura 2).

En el AC, el método elegido por el coeficiente de correlación cofenética $(0,728)$ fue encadenamiento promedio o UPGMA (sus siglas en inglés) y el número de conglomerados seleccionados fue 4 (figura 3). El primer conglomerado ( $21 \%$ del total de ovinos) está conformado exclusivamente por ovinos de BA, cap-

Tabla IV. Coeficiente de correlación entre las variables originales y los 2 primeros componentes principales (Correlation coefficient between the original variables and the first two principal components).

\begin{tabular}{lrr}
\hline Variables & CP 1 & CP 2 \\
\hline DMF & 0,94 & $-0,27$ \\
DE_DMF & 0,85 & 0,36 \\
CU & $-0,91$ & 0,08 \\
FC & $-0,87$ & 0,43 \\
LM & 0,46 & 0,84
\end{tabular}

$\mathrm{DMF}=$ Diámetro medio de fibra; DE_DMF= Desvío estándar del diámetro medio de fibra; $\mathrm{CU}=$ Curvatura de ondulación; FC= Factor de confort; LM= Largo de mecha. 


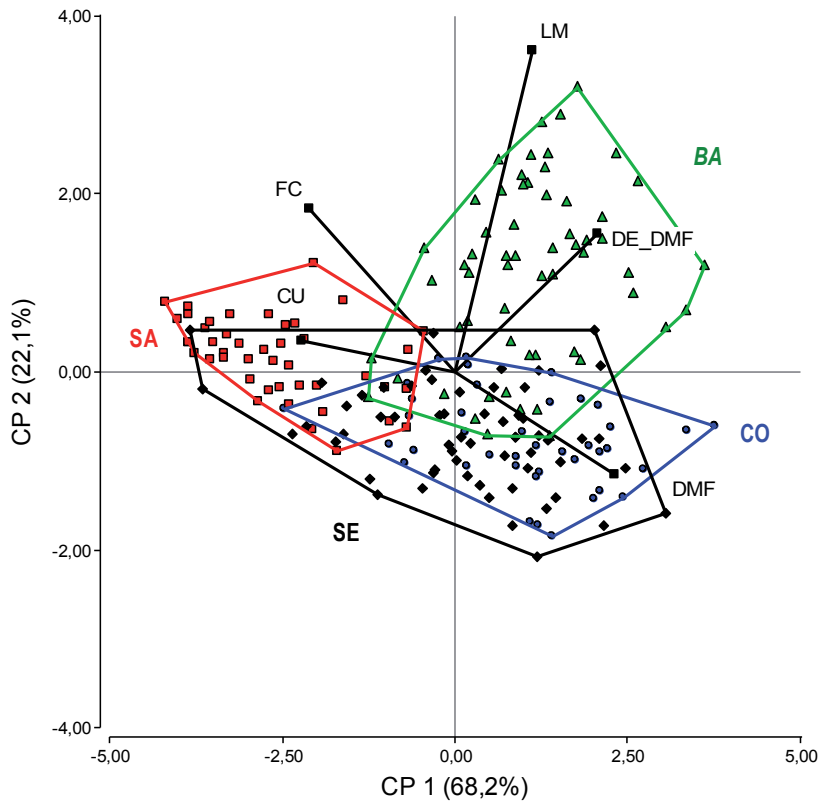

$\triangle \mathrm{BA}=$ Buenos Aires; $-\mathrm{SA}=$ Salta; $\diamond \mathrm{SE}=$ Santiago del Estero; $\bullet \mathrm{CO}=$ Corrientes.

Figura 2. Biplot de las características de la lana y ovinos según la región (Biplot of wool characteristics and sheep sorted by region).

Tabla V. Valores medios y desvíos estándar para las cinco variables en cada una de los cuatro conglomerados (Mean values and standard deviations for the five variables in every one of the four clusters).

\begin{tabular}{lcccc}
\hline \multicolumn{5}{c}{ Región } \\
\hline Variable & $1(n=43)$ & $2(n=6)$ & $3(n=112)$ & $4(n=42)$ \\
\hline DMF $(\mu m)$ & $33,1 \pm 2,3$ & $42,5 \pm 2,2$ & $33,9 \pm 3,4$ & $25,4 \pm 1,9$ \\
DE_DMF $(\mu \mathrm{m})$ & $11,8 \pm 1,7$ & $14,0 \pm 0,8$ & $9,2 \pm 2,0$ & $5,6 \pm 1,0$ \\
CU $(\% / m m)$ & $42,0 \pm 6,5$ & $28,1 \pm 5,1$ & $44,4 \pm 11,3$ & $81,5 \pm 12,1$ \\
FC $(\%)$ & $52,2 \pm 12,6$ & $21,1 \pm 5,8$ & $42,4 \pm 14,9$ & $82,4 \pm 9,3$ \\
LM $(\mathrm{mm})$ & $137,8 \pm 27,2$ & $99,3 \pm 50,3$ & $52,3 \pm 16,5$ & $47,6 \pm 14,5$ \\
\hline
\end{tabular}

$\mathrm{DMF}=$ Diámetro medio de fibra; DE_DMF= Desvío estándar del diámetro medio de fibra; $\mathrm{CU}=$ Curvatura de ondulación; $\mathrm{FC}=$ Factor de confort; LM= Largo de mecha.

turando el $73 \%$ del total de esa región. Se caracteriza por tener el mayor LM promedio (tabla V). El segundo conglomerado es irrelevante debido a que representa el 3\% del total de ovinos (6 animales). El tercer conglomerado (55\% de los animales), está conformado principalmente por las regiones SE (48\%) y CO (33\%), aportando la primera el $90 \%$ de sus ovinos y $\mathrm{CO}$ el $93 \%$. El cuarto conglomerado (21\% del total) básicamente se conforma por ovinos de SA (86\%), representando el $82 \%$ de los ovinos de esa región. Este último conglomerado presenta los mayores promedios en CU y FC y los menores promedios en las restantes variables medidas (tabla V).

Dado la presencia de heterogeneidad de varianzas entre los grupos, se empleó análisis discriminante cuadrático en lugar de lineal. Al utilizar las cuatro regiones no resulta adecuada pues la tasa de error fue del $21 \%$. En cambio si se juntan CO y SE (en base a los resulta-

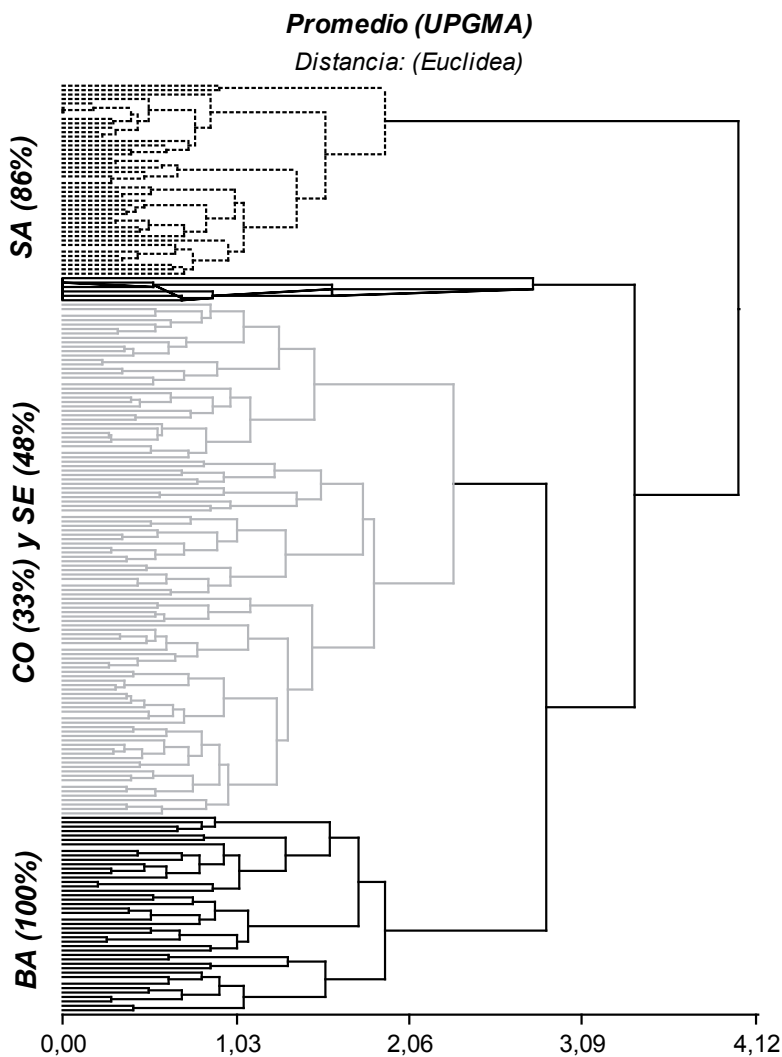

Figura 3. Dendrograma construido según el método UPGMA que identifica 3 grupos donde se indica el porcentaje de preponderancia de cada región (Dendrogram constructed according to the UPGMA method which identifies three groups in which the prevalence rate for each region is shown).

dos del AC) la tasa se redujo al 11\%, no superando en cada una de las tres regiones el 13\% (el 9\% en SA, el $12 \%$ en BA, y el 13\% en la nueva región CO-SE).

Ninguna de las regiones muestra promedios de DMF superiores a los $40 \mu \mathrm{m}$ o más, como se establece en la clasificación lanera argentina para ser considerada como lana para alfombras, carpet wool o Criolla (Calvo, 1983; Aguirre et al., 2010). Se observa que el promedio del DMF de las ovejas de las cuatro regiones se ubican en clasificaciones de finura que van desde cruza fina (SA), cruza mediana (SE y BA) y cruza gruesa (CO) que corresponden a las razas Corriedale, Romney Marsh y Lincoln respectivamente. Por ejemplo el DMF en ovejas Corriedale en Perú, fue estimado en 26,06 $\pm 5,84$ um (Guzman Barzola et al., 2010); en la región mesopotámica para la misma raza, los indicadores productivos son bajos y predominan lanas entre 25 y $32 \mu$ de diámetro (Gambetta y Pueyo, 2004) y en la localidad de Monte Caseros provincia de Corrientes Argentina en 29,4 $\mu \mathrm{m}$ (Flores Quintana et al., 2012), valores similares a los observados en el grupo SA. Para las razas doble propósito Romney Marsh, Coopworth, Perendale y Texel, en Nueva Zelandia el DMF, teniendo en cuenta 1271 observaciones fue estimado en 33,30 $\pm 2,54 \mu \mathrm{m}$

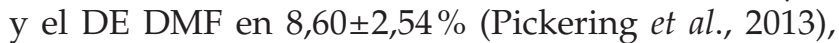
valores similares a los obtenidos por las ovejas Criollas de los grupos SE y BA. Los resultados de DMF obtenidos, son concordantes con los de la oveja Criolla 
formoseña que registraron una media de $26,9 \pm 8,6 \mu \mathrm{m}$ para los animales de color café y de 31 a $33 \mu \mathrm{m}$ para los fenotipos blanco, gris y negro (Galdamez et al., 2012) y también con los reportados para la oveja Linca donde se obtuvo una media para el $\mathrm{DMF}=27,5 \pm 4,3 \mu \mathrm{m}$ (Reising et al., 2008), lana que de acuerdo a estos valores, tampoco puede ser clasificada como carpet wool o lana de alfombras. La CU está directamente relacionada con la ondulación de la lana y las lanas con ondulaciones bien marcadas son aquellas que tienen buen carácter y pueden clasificarse en baja curvatura (valores menores a $50 \% \mathrm{~mm}$ ); media curvatura (valores de 60 a $90^{\circ} / \mathrm{mm}$ ) y de alta curvatura (valores mayores a $100 \% / \mathrm{mm}$ ). Lanas cruzas de $30 \mu$ m o más, generalmente tendrán baja curvatura, la lana Merino típica una curvatura media y las Merino superfina una curvatura alta (Sacchero, 2008). Los datos obtenidos para los cuatro grupos de ovejas Criollas revelan que la CU del grupo SA presenta una Curvatura Mediana, compatible con una lana fina, mientras que SE, BA y CO una Curvatura Baja, compatible con una lana Cruza. El FC de las ovejas de Salta resultó ser mucho mayor que en el resto de los grupos, lo cual indica que tiene una proporción significativamente mayor de fibras menores de $30 \mu \mathrm{m}$ que SE, CO y BA. El LM es una característica de gran importancia desde el punto de vista textil, ya que si las fibras son largas ( $>50 \mathrm{~mm}$ ) se utilizan para el sistema de peinado y si son cortas $(<50 \mathrm{~mm})$ se utilizan en el sistema de cardado (Guzman et al., 2010). Solamente BA y CO superan los $50 \mathrm{~mm}$ de $\mathrm{LM}$, siendo BA= 123 $\mathrm{mm}$, significativamente mayor al resto de los grupos

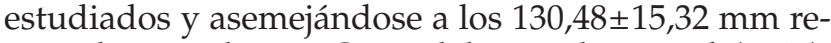
portados para la raza Corriedale por Flores et al. (2012).

\section{CONCLUSIONES}

En base a los resultados obtenidos por sus características laneras, han quedado definidos tres grupos de ovejas correspondientes a las regiones de: a) Salta (SA); b) Buenos Aires (BA) y c) Corrientes y Santiago del Estero (CO-SE). La mayor uniformidad y mejor calidad de lana en cuanto a finura, factor de confort y curvatura de ondulación del grupo de ovejas salteñas respecto de los otros dos, se atribuye a la evolución de ese genotipo restringido a las condiciones ambientales de la región y al manejo selectivo realizado por sus criadores. Ninguno de los tres grupos puede clasificarse en la categoría de lana carpet-wool o Criolla o de alfombras según la clasificación lanera argentina. Se considera una tarea de gran valor zootécnico la de ampliar el estudio de las características laneras de los ovinos Criollos y extenderlo a las poblaciones adaptadas a las distintas regiones, de tal forma de ir conformando un mapa lanero Criollo de la República Argentina, para favorecer el uso racional de este recurso zoogenético.

\section{AGRADECIMIENTOS}

A los señores: Fortunato Baños y César Herrera, Iruya, Salta; Ariel Loto, El Pirucho, Santiago del Estero; Enrique Bouillon, Sauce, Corrientes; Miguel Berretta, 25 de Mayo, Buenos Aires; que mantienen majadas de este valioso recurso zoogenético y nos permitieron realizar los muestreos correspondientes.

\section{BIBLIOGRAFÍA}

Aguirre, A. y Fernández, R. 2010. Manual de acondicionamiento de lanas. 2nd versión. Prolana. Ministerio de Agricultura Ganadería y Pesca. Argentina. 73 pp.

Baxter, P. 2001 a. Precision of measurement of diameter, and diameterlength profile, of greasy wool staples on-farm, using the OFDA2000 instrument. Wool Tech Sheep Bree, 49: 42-52.

Baxter, P. $2001 \mathrm{~b}$. On-farm classing of animals and fleeces with the OFDA2000. Wool Tech Sheep Bree, 49: 133-155.

Calvo, C. 1983. Ovinos: Ecología. Editor Massiero Hnos. Buenos Aires, Argentina. 299 pp.

Cardinaletti, L.; Von Thungen, J. y Lanari, M.R. 2011. Comercialización de artesanías fabricadas con lana de ovejas linca en la Patagonia Argentina. Añadiendo valor a la diversidad ganadera. FAOProducción y Sanidad Animal. № 168. $156 \mathrm{pp}$

Carrazzoni, J.A. 1997. Crónicas del campo argentino, nuestras raíces agropecuarias. Academia Nacional de Agronomía y Veterinaria. Orientación Gráfica Editora SRL. Buenos Aires. 405 pp.

Cuadras, C.M. 2014. Nuevos métodos de análisis multivariante. Barcelona: CMCEditions. España. http://www.ub.edu/stat/personal/ cuadras/metodos.pdf (30/03/2014).

Di Rienzo, J.A.; Casanoves, F.; Balzarini, M.G.; Gonzalez, L.; Tablada, M. y Robledo, C.W. 2012. InfoStat versión 2012. Grupo InfoStat, FCA. Universidad Nacional de Córdoba. Argentina. http://www. infostat.com.ar (03/05/2012).

Flores Quintana, C.; Yañez, E.; Carlino, M. y Bangher, G. 2012. Morfología de la piel y producción de lana en cruzamiento absorbente con Merino multipropósito. J Morphol, 30: 1434-1441.

Galdámez, D.; De la Rosa, S.; Perezgrovas, R.; Revidatti, M.A.y Rodríguez G. 2012. Características macroscópicas y microscópicas de la mecha y la fibra de lana en la oveja autóctona Formosa de Argentina. Actas Iberoamericanas de Conservación Animal. AICA, 2: 309-312.

Gambetta, R. y Pueyo, J. 2004. Producción ovina en la Mesopotamia Argentina. Idia XXI. 4:16-21.

Guzmán Barzola, J.C.y Aliaga Gutiérrez, J. 2010. Evaluación del método de clasificación del vellón en ovino Corriedale (ovis aries) en la Sais Pachacutec. Facultad de Zootecnia Universidad Nacional Agraria La Molina. www.produccion-animal.com.ar (22/05/2012).

Helman, M. 1951. Ovinotecnia. Tomo 1. Editorial El Ateneo. Buenos Aires. $674 \mathrm{pp}$.

Lembeye, F.; Castellaro, G.; Magofke, J.C. y Uribe, H. 2014. Correlaciones fenotípicas entre características de importancia económica en ovinos Suffolk Down y Merino Precoz en la zona central de Chile. Archiv Med Vet, 46: 103-109.

Mc Culloch, C.E. and Searle, S.R. 2001. Generalized, linear and mixed models. John Wiley and Sons. New York. USA. 325 pp.

Mueller, J.P. 2006. Avances en el mejoramiento genético de ovinos en la Argentina. Comunicación técnica INTA EEA Bariloche Nro. PA 493. $6 \mathrm{pp}$

Peña, D. 2002. Análisis de datos multivariantes. McGraw-Hill/Interamericana de España. S.A.U. Madrid. 539 pp.

Pérezgrovas Garza, R. y Parés Casanova, P.M. 2013. Razas autóctonas de ganado lanar en lberoamérica. Desarrollo histórico y características de la lana. Universidad Autónoma de Chiapas. México. 435 pp.

Peterson, A.D. and Gherardi, S.G. 2001. The ability of the OFDA2000 to measure fleeces and sale lots on-farm. Wool Tech Sheep Bree, 49: 110-132.

Pickering, N.K.; Blair, H.T.; Hickson, R.E.; Dodds, K.G.; Johnson, P.L. and McEwan, J.C. 2013. Genetic relashionships between dagginess, breech bareness, and wool traits in New Zeland dual-purpose sheep. J Anim Sci, 91: 4578-4588.

Reising, C.; Maurino, M.J.; Basualdo, A. y Lanari M.R. 2008. Calidad de lana de la oveja Linca en el noroeste de la Patagonia. Memorias del IX Simposio Iberoamericano de Recursos Genéticos. Mar del Plata. Tomo II: 397-400. 
Sacchero, D. 2008. Utilización de medidas objetivas en fibras textiles para determinar calidad. En: Biotecnología aplicada en camélidos sudamericanos. Ed. Edgar Quispe Peña Huancavelica. Perú. pp. 37-65.

Safari, E.; Fogarty, N.M. and Gilmour, A.R. 2005. A review of genetic parameter estimates for wool, growth, meat and reproduction traits in sheep. Livest Prod Sci, 92: 271-289.

SAS Institute Inc. 2009. SAS OnlineDoc $\circledR^{9}$ 9.2. Cary, NC: SAS Institute Inc. Cary, NC. USA.

Sokal, R.R. and Rohlf, F.J. 1962. The comparison of dendrograms by objective methods. Taxon, 11: 33-40. http://www.jstor.org/ stable/1217208? seq=1\#page_scan_tab_contents (17/12/2008).
Sponenberg, D.P. and Taylor, C. 2009. Navajo-Churro sheep and wool in the United States. Animal Genetic Resources Information () Food and Agriculture Organization of the United Nations. 45: 99-105.

Steinhagen, O. and de Wet, P.J. 1986. Phenotypic correlations between wool traits of a Dohne Merino flock. S Afr J Anim Sci, 16: 99-100.

Vaillemans, B.A. 2003. Calidad de la lana de ovinos Corriedale en la zona húmeda de la XII Región: Efecto del hibridaje con líneas paternales Texel. Tesina. Pontifica Univ. Católica de Chile Facultad de Agronomía e lng. Forestal. $50 \mathrm{pp}$.

Zeballos, E. 1898. Descripción amena de la República Argentina. Tomo III. A través de las cabañas. Editor Jacobo Peuser. La Plata. Argentina. 
